\title{
Vagabond: a new project for macromolecular model refinement.
}

Helen Ginn, Diamond Light Source Ltd.

Model refinement for biomolecular crystallography, at present, relies on a model defined in atomic $\mathrm{x}, \mathrm{y}, \mathrm{z}$ parameters and associated $\mathrm{B}$ factors. Vagabond is a new refinement project which revisits the concept of refining in torsion space, defining the model in terms of bond lengths, angles and torsion angles. This significantly reduces the number of parameters required to describe most of the structure. However, it also incorporates a novel model for expressing flexibility in a chemically sensible manner, and is capable of describing weird and wonderful atomic distributions which are not accessible from isotropic or anisotropic B factor models. The combination of these features leads to a reduction in overfitting and increased clarity of maps. 